\title{
De la Vie à la Mort. La réception de La Sépulture de Gabriel Marie Legouvé en Espagne et sa traduction par Manuel Norberto Pérez de Camino
}

\author{
Giorgia MARANGON \\ Departamento de Ciencias del Lenguaje \\ Universidad de Córdoba \\ lrlmarmg@uco.es
}

\begin{abstract}
RESUMÉ
Le 6 octobre 1797 à Paris, qui se remettait à peine de la féroce passion révolutionnaire, Gabriel-Marie Legouvé, membre éminent de l'Institut National, lisait son poème intitulé «La Sépulture». Lors de sa publication dans les Mémoires de l'Institut National seulement quatre ans après sa date de composition, son auteur y ajoute la note suivante : «C'est l'indécence avec laquelle on inhume aujourd'hui que j'attaque dans ses vers, où je rappelle la profanation des tombeaux. Je ne la crois pas étrangère au sujet, puisqu'elle est la première outrage fait à la dignité de l'homme et au respect qu'on doit aux morts». Ce furent donc les débats qui surgirent en France entre 1795 et 1804 sur la manière de mener à bout les enterrements ce qui tissa la toile de fond sur laquelle G.-M. Legouvé se mit à travailler. Son ouvrage n'atteignit cependant l'Espagne qu'en 1822, vingt et un ans après sa parution, grâce à la traduction que Manuel N. Pérez de Camino en a faite. Et c'est justement cette version qui en constitue la première et peut-être la seule trace en Espagne, et par là même une référence importante pour les spécialistes de ce genre littéraire.
\end{abstract}

Mots clé: élégie, sépulture, philologie, traduction, France, Espagne

[Recibido, septiembre 2013; aprobado, diciembre 2013]

De la vida a la muerte. La recepción de Le Sépulture de Gabriel Marie Legouvé en España y la traducción por Manuel Norberto Pérez de Camino

\begin{abstract}
RESUMEN
El 6 de octubre de 1797, en un París que se estaba recuperando de la cruenta fiebre revolucionaria, un ilustre miembro del Instituto Nacional, Gabriel-Marie Legouvé, leía un poema titulado La Sépulture. Publicado sólo cuatro años más tarde de su fecha de composición en las Mèmoires de l'Institut Nacional, el autor le añadía la siguiente nota: "C'est l'indécence avec laquelle on inhume aujourd'hui que j'attaque dans ses vers, où je rappelle la profanation des tombeaux. Je ne la crois pas étrangère au sujet, puisqu'elle est la première outrage fait à la dignité de l'homme et au respect qu'on doit aux morts». Los debates surgidos en Francia entre 1795 y 1804 sobre la manera de enterrar constituyen el fondo sobre el que empieza a trabajar Gabriel-Marie Legouvé. La obra del francés llega a España traducida por Manuel N. Pérez de Camino en 1822, veintiuno años después de su publicación. Es el primer y único testimonio de la elegía de Legouvé en España y un importante referente para los estudiosos de este género literario.
\end{abstract}

Palabras clave: elegía, sepultura, filología, traducción, Francia, España 


\section{Introduction}

Le 6 octobre 1797, dans un Paris qui se relève de la sanglante fièvre révolutionnaire, un membre éminent de l'Institut National, Gabriel-Marie Legouvé, lisait un poème intitulé La Sépulture (Legouvé 1801: 180-193). Publié quatre ans seulement après sa date de composition, en 1801, dans les Mémoires de l'Institut National, il porte la note suivante, ajoutée par l'auteur: «C'est l'indécence avec laquelle on inhume aujourd'hui que j'attaque dans ses vers, où je rappelle la profanation des tombeaux. Je ne la crois pas étrangère au sujet, puisqu'elle est le premier outrage fait à la dignité de l'homme et au respect qu'on doit aux morts» (Legouvé 1801: 181).

Les débats qui ont porté en France entre 1795 et 1804 sur la manière d'enterrer forment la toile de fond sur laquelle Gabriel-Marie Legouvé commence à travailler. Cet auteur n'a fait que reprendre et traduire en un discours poétique tout ce qui, à cette époque, était débattu avec ferveur et esprit polémique dans les revues et les journaux, faisant ainsi de la France l'un des pays qui se sont intéressés le plus au genre littéraire sépulcral.

Pendant toute la deuxième moitié du XVIIIème siècle, la poésie sépulcrale fut un phénomène littéraire et culturel important en Europe, comme le fut aussi et tout autant la question de l'hygiène des tombeaux, puisque la dénommée "Police Médicale" proposait d'enterrer les morts à l'intérieur ou à l'extérieur de la ceinture urbaine, dans les églises paroissiales ou dans les cimetières publics. Le sujet de la mort, étroitement lié à la sépulture, s'est toujours situé au premier plan dans l'histoire européenne. Les œuvres littéraires consacrées à ce sujet sont le reflet d'un processus historique et juridique qui occupa une place centrale dans la société de l'époque et à toutes les époques. Parmi les textes les plus importants de droit funéraire (Marantonio Sguerzo 1976: 1-345 ; Tolívar 1983: 1-254 ; Pérez Gálvez 1997: 1-443), il est extraordinaire de constater à quel point, depuis les temps les plus anciens, le sujet de la mort et les mesures adoptées à cet égard, marquent le début de la civilisation. Religion, littérature, histoire et droit forment l'assise du développement si large et élaboré qu'a eu le thème de l'enterrement au long des siècles. La religion est le moteur qui meut ce processus, la littérature et l'histoire le communiquent et le droit le régit et l'ordonne.

Depuis la loi des Douze Tables, fondement du droit funéraire romain, qui disposait qu'aucun cadavre ne pouvait être enterré ou brûlé à l'intérieur de la ville (hominem mortuum in urbe ne sepelito, neve urito - Cicerone, De Leg. Lib. $2 \mathrm{n}^{\circ} 25$ e 26), jusqu'à l'Édit napoléonien de Saint-Cloud 1804, en passant par les nombreux édits et conciles qui réglementaient les enterrements, l'importance du sujet de la mort et de la sépulture en Europe n'a cessé de croître.

L'importance de la littérature consacrée au thème sépulcral doit en grande part sa force à des auteurs qui, doués d'extraordinaires capacités artistiques, ont su faire de l'histoire un prétexte pour exploiter leurs thèses dans un registre littéraire. Le prétexte historique de Gabriel Marie Legouvé fût la profanation des tombeaux dans une grande nécropole royale : l'abbaye de Saint-Denis à Paris. L'histoire narre que, dans le but de fêter la prise des Tuileries le 10 août 1793, les révolutionnaires ont voulu détruire les tombes des monarques et jeter les corps exhumés dans une fosse commune (où ceux-ci sont restés jusqu'au règne de Louis XVIII - en 1817 -, qui ordonna la restitution des restes à la basilique de Saint-Denis, où Louis XVI et Marie Antoinette ont également été inhumés). Pendant ces sombres jours de 1793, Dom Poirier, savant bénédictin de l'abbaye de Saint-Denis, avait été nommé « commissaire politique », avec pour mission d'assister à l'exhumation, dans un bon nombre de tombes, de princes et de rois réduits en poussière ou en voie de putréfaction. Avec la 
Révolution, des tombes de grands personnages de l'histoire politique et militaire française furent également profanées ; parmi ces noms célèbres, G. M. Legouvé cite dans son poème : Henri de La Tour d'Auvergne - Bouillon (Vicomte de Turenne) et Bertrand Du Guesclin.

\section{La Sépulture de Gabriel Marie Legouvé et ses répercussions en Espagne}

Gabriel Marie Legouvé publie La Sépulture en 1801 sous la forme d'une élégie. Il s'agit de 160 vers dans lesquels l'auteur français dénonce un moment historique bien déterminé : l'époque postrévolutionnaire, également appelée la Terreur ; il s'en prend à ceux qui n'ont pas respecté le repos et la mémoire des hommes illustres, en violant leurs tombes et en répandant leurs cendres à tous les vents.

Chargée d'un lyrisme patriotique, l'élégie de Legouvé ouvre en France les débats sur l'utilité de la sépulture pour les vivants, pour l'État et pour la société dans son ensemble. Les tombes des grands, où se reconnaît l'esprit d'un peuple, permettent de faire revivre dans cette grandeur l'auspice d'une libération future. C'est la raison pour laquelle Legouvé livre une lutte sans merci à l'utilisation fréquente des fosses communes à l'époque révolutionnaire, parce qu'il est ignominieux de penser que «auprès de scélérats gît l'homme vertueux» (v. 116). C'est un hymne au respect dévasté par la fureur de la révolution: la profanation et la violation des tombes et l'utilisation des fosses communes où se mêlent les ossements du juste à ceux du méchant «du juste qui n'est plus, respectez le repos ; / du juste et des méchants, séparez les tombeaux» (vv. 119-120). C'est un hymne à la mémoire pour ne pas oublier ce qui est arrivé : même le plus sauvage couvrait de terre les morts pour les protéger des intempéries et de la férocité des animaux : «le sauvage lui-même, inhumain, implacable / toujours d'un peu de terre a couvert son semblable», tandis que la moderne France a oublié la dignité et le respect que l'on devait aux morts. La douleur d'un frère ou les larmes d'un fils suffisent pour ne pas laisser tomber dans l'oubli la mémoire du défunt «Mais appelez, du moins, autour de nos débris / et la douleur d'un frère, et les larmes d'un fils» (v. 107-108). Et pour finir, c'est un hymne à la simplicité évoquée par l'auteur en exprimant son souhait d'être enterré sous un arbre entouré de fleurs, dans la paix du locus amoenus : «Lorsqu'un mortel n'est plus, là, les siens, près du temple, / vont déposer sa cendre en un bocage épais; / y plantent des lilas, des roses, des œillets, / arrosent chaque jour leurs tiges abreuvées (vv. 142-145)».

Dans les pays de langue romane, l'histoire de la poésie nocturne et sépulcrale présente des caractéristiques particulières. À quelques rares exceptions près, tout ce qu'on connaît dans ces pays des prototypes de ce genre littéraire se résume aux traductions françaises que Pierre Le Tourneur offre d'Edward Young, James Hervey et Thomas Gray, les précurseurs, à tous les effets, de ce type de poésie. Notamment dans l'Élégie de Gray : The Elegy written in country churchyard (1716-1771), on remarque le goût pour les jardins anglais et pour la mélancolie, sujets chers à Legouvé qui devait à Gray le cadre des cimetières anglais, mais qui ajoute à son poème un sentiment national et patriote totalement inconnu de Gray.

En Espagne comme en France, la poésie lugubre et sépulcrale vient directement, non pas des textes anglais de Young, Hervey et Gray, mais des traductions qui sont apparues des travaux de ces auteurs.

La Sépulture de Legouvé arrive en Espagne traduite par Manuel Norberto Pérez de Camino en 1822, vingt-et-un ans après sa parution en France. C'est le premier et unique témoignage de l'élégie de Legouvé en terre ibérique et une très importante référence pour les adeptes de ce genre littéraire. Au travers la plume d'un «afrancesado» (écrivain «franci- 
sé»), comme l'appelle la critique contemporaine, arrive en Espagne une des œuvres les plus représentatives de la lyrique sépulcrale européenne : El mérito de las mujeres, los recuerdos, la sepultura, la melancolía : poemas de Gabriel Legouvé, traducidos en versos castellanos por Don M. N. Pérez de Camino (1822: 255-293).

L'œuvre de Manuel N. Pérez de Camino est fondamentalement littéraire et traductologique. Son œuvre principale est une Poética éditée en 1829 à Bordeaux. Son œuvre de poète nous parvient par la Biblioteca de Autores Españoles (Rivadeneyra 1869-1875: 721-722) et par les commentaires que Marcelino Menéndez Pelayo lui consacre dans l'Historia de las ideas estéticas en España (1965: 28), mais c'est Alonso Martínez, économiste et parent de Camino, qui nous le fait connaitre en tant que traducteur, en publiant, entre les années 1874 et 1878, toutes ses traductions restées en possession de la famille Pérez de Camino après sa mort. Pendant ses années d'exil en France, il se consacre à la traduction et, même si son admiration pour la littérature classique le porte à traduire des poètes latins, sa traduction du français des poèmes de Gabriel Legouvé est sublime. Elle est et reste le seul témoignage de l'influence de celui-ci en Espagne jusqu'à nos jours.

\section{La traduction de La Sépulture, de Manuel Norberto Pérez de Camino}

Richiede qualche rinforzo di cautele la lettura di testi lirici tradotti. Se già numerosi sono i diaframmi che si frappongono tra il testo lirico e la fruizione di esso da parte del comune lettore, tanto più si accentua il divario quando ci si accosta a un testo tradotto. Quello che in apparenza sembra un semplice mutamento del codice linguistico comporta in realtà una trasposizione che investe globalmente la mentalità, la Weltanschauung, l'esperienza storica, insomma la civiltà che sottostà al testo (Roncoroni 1999: 304).

La traduction par M. N. Pérez de Camino de La Sépulture observe les critères traductologiques que nous trouvons dans la préface de sa traduction des Elegías de Tíbulo; les lignes directrices qui le guideront dans ses travaux de traduction seront les suivantes:

Fidelidad sin servidumbre, concisión en cuanto la claridad y el artificio métrico lo han permitido. Imitación, sin licencia, de imágenes, de armonía y sobre todo de expresión, parte la más esencial, la más difícil de verter, y sin la cual es imposible dar una idea del poeta que se traduce, especialmente en esas poesías fugitivas en que la expresión forma el mérito principal (Alonso Martínez 1874: 13).

Dans la préface de la traduction de l'œuvre de Legouvé, Pérez de Camino expose les motifs de son travail (1822: 12-17):

Cuando un pueblo, largo tiempo abismado en un letargo moral, indignado de su abatimiento, hace un esfuerzo sobre sí mismo y ambicionando la elevación, á que le llaman sus brillantes calidades, abre su alma á los altos pensamientos y su pecho á los sentimientos generosos, ofrecerle producciones literarias, que alimenten en él esta sensibilidad delicada, fuente de todos los progresos del espíritu, de toda gloria y de toda virtud, es darle medios para facilitarle la grande empresa de su reforma. [...] Este pueblo es la España: y un hijo suyo cree poder contribuir á facilitarla todas las ventajas referidas, publicando la presente traducción de las poesías escogidas de Legouvé. Dictadas estas poesías por el corazón, despiertan en el lector una sensibilidad exquisita; modelos fáciles de gusto, pueden servir poderosamente á inspirar en todas las clases el de la literatura; llenas de bellas imágenes y de pinturas deliciosas, hacen olvidar los ciudadanos y las penas, con una distracción agradable. Legouvé juntaba á una alma delicadísima un gusto perfecto. Sucesor de Delille en la cátedra de poética, lo fue también de la elegancia, de la armonía y de la sensibilidad del Virgilio francés, y estas gracias y el candor y amenidad, que respiran sus versos, le han merecido el epíteto de amable. [...] Su panegírico del sexo hermoso es una obra maestra. La buena lógica y la poesía rivalizan en él, para vengar á esta preciosa mi- 
tad del género humano, injustamente denigrada. [...] El poema de los Recuerdos es una galería mágica, en la cual el lector pasa de cuadros en cuadros, á cual más instructivos, á cual más agradable y acabados. El de la Sepultura es una elegante lección de filosofía y humanidad, y en el de la Melancolía, el poeta ha esparcido el claro obscuro tierno, patético, que tanto conviene a este sentimiento.

Le poème de 312 vers que nous analysons ici est La Sepultura. Il y a lieu de remarquer que, parmi les notes au poème, il y a une Elegía escrita sobre el cementerio de una aldea, qui est une imitation de la célèbre composition du poète anglais Thomas Gray, traduite en plusieurs langues.

\subsection{Comparaison textualle}

\begin{tabular}{|c|c|c|}
\hline La Sépulture & & La Sepultura \\
\hline Où sont ces vieux tombeaux et ces marbres antiques & & ¿ Do lo mármoles antiguos, \\
\hline Qui des temples sacrés décoraient les portiques? & & Do las viejas tumbas se hallan, \\
\hline & & Que de nuestros sacros templos \\
\hline Viola des prisons l'asile épouvanté, & & Los pórticos decoraban? \\
\hline Coururent, tout sanglants, de nos aïeux célèbres & 5 & ¡O crimen! esos malvados \\
\hline Profaner, mutiler les monuments funèbres, & & $\begin{array}{l}\text { Cuya furibunda audacia, } \\
\text { Violó las prisiones }\end{array}$ \\
\hline Et commettre, à la voix d'un lâche tribunat, & & La mansión amedrentada, \\
\hline Sur des cadavres même un autre assassinat! & & Corren, y de ilustres nombres, \\
\hline Gloire, talents, vertus, rien n'arrêta leur rage. & & Con manos ensangrentadas, \\
\hline O guerriers généreux, dont le mâle courage & 10 & Los fúnebres monumentos \\
\hline De l'État ébranlé releva le destin, & & Despedazan y profanan, \\
\hline Vengeurs du nom français, Turenne, Du Guesclin, & & Y cometen, de tribunos \\
\hline Vous vîtes par leurs mains vos cendres dispersées & & $\begin{array}{l}\text { Vles a la voz infausta, } \\
\text { Un asesinato nuevo }\end{array}$ \\
\hline Errer au gré des vents, de vos urnes chassées! & & Sobre cenizas heladas. \\
\hline La beauté ne put même adoucir leur courroux ; & 15 & Gloria, talentos, virtudes \\
\hline Sévigné, dans la mort tu ressentis leurs coups ! & & Nada detiene su rabia. \\
\hline C'en est donc fait ! brisant les tombes révérées, & & O generosos guerreros, \\
\hline Ils ont désenchanté nos enceintes sacrées : & & Cuyas valientes espadas, \\
\hline Nous y cherchons en vain ces marbres inspirants & & Del estado aseguraron \\
\hline Où nos yeux se plaisaient à s'arrêter longtemps, & 20 & La suerte mal afirmada, \\
\hline Où nos cœurs admiraient, épris de leur histoire, & & $\begin{array}{l}\text { Turena, o du Guesclin, } \\
\text { Vengadores de la Francia, }\end{array}$ \\
\hline Les dons de la patrie et les bruits de la gloire, & & Mirasteis vuestras cenizas, \\
\hline Et sur l'affreuse mort, dont tout est dévoré, & & Por sus manos dispersadas, \\
\hline Des talents, des vertus le triomphe assuré. & & Juguete del viento, expulsas \\
\hline On se sent agrandir au tombeau d'un grand homme ! & 25 & De su fúnebre morada. \\
\hline Les arts m'en sont garants; des morts que l'on renomme & & La beldad misma no pudo \\
\hline Dans le bronze vivant, dans le marbre animé, & & Ablandar fiereza tanta, \\
\hline Ils rendront tous les traits à l'univers charmé. & & En la muerte, o Sevigné, \\
\hline Mais ce n'est point assez pour le cœur qui les aime, & & Probaste su cruda saña. \\
\hline Leurs images, hélas ! Ne seront point eux-mêmes ! & 30 & $\begin{array}{l}\text { Triunfan doquier : demoliendo } \\
\text { Estas tumbas veneradas, }\end{array}$ \\
\hline C'est eux ou leurs débris que nous voulons trouver: & & Fieros han desencantado \\
\hline Au pied de leurs tombeaux nous aimons à rêver. & & Nuestras mansiones sagradas. \\
\hline Là, du recueillement savourant tous les charmes, & & Los mármoles inspirantes \\
\hline Nous trouvions à-la-fois des leçons et des larmes ; & & Buscan en vano mis ansias, \\
\hline Il semblait que du fond de ces cercueils fameux & 35 & Do fijarse largo tiempo \\
\hline Une voix nous criât : « Illustrez-vous comme eux !» & & Gustaban nuestras miradas ; \\
\hline Voilà l'illusion que nous avons perdue. & & Do, embebidos en su historia, \\
\hline Vous tous, que pleure encor la patrie éperdue, & & Nuestros pechos admiraban \\
\hline Consolez-vous pourtant si vos corps mutilés & & De la gloria los derechos \\
\hline Loin de leurs monuments languissent exilés : & 40 & Y sobre la horrible muerte, \\
\hline Bannis de vos cercueils, et non de votre gloire, & & Que todo avara lo traga, \\
\hline Vous restez dans nos cœurs et dans notre mémoire. & & De la virtud y el ingenio \\
\hline Là, se sont retranchés vos débris immortels ; & & La victoria asegurada. \\
\hline Là, se sont relevés vos tombeaux, vos autels ; & & En la tumba de un grande hombre \\
\hline
\end{tabular}


Et contre les pervers soulevant tous les âges,

Vous immortalisez jusqu'à leurs vils outrages.

Mais de quel crime encor mon œil est révolté !

Par des bras soudoyés un cadavre porté,

Sans cortège, sans deuil, s'avance solitaire ;

C'est ainsi parmi nous qu'on rend l'homme à la terre !

Autrefois l'amitié, la nature, et l'amour,

Accompagnant sa cendre à ce dernier séjour,

Lui portaient en tribut leur douleur consolante :

Maintenant, inhumé sans la pompe touchante

Qui suivait le mortel dans la tombe endormi,

On dirait qu'il n'eut pas un parent, un ami !

A-t-il perdu ses droits en perdant la lumière?

N'est-il point un respect qu'on doive à sa poussière ?

Sur les rives du Nil un zèle industrieux,

Par un baume éternel, perpétuant aux yeux

Une mère expirée, une épouse ravie,

Savait tromper la mort ou figurer la vie ;

Les Grecs et les Romains présentaient aux tombeaux

Des offrandes, des pleurs, et le sang des taureaux ;

Le sauvage lui-même, inhumain, implacable,

Toujours d'un peu de terre a couvert son semblable ;

Et vous, peuple poli, dans cet âge si beau,

Où Montesquieu, Voltaire, et Raynal et Rousseau,

Par leurs savants écrits, pleins d'Athènes et de Rome,

Apprirent aux humains la dignité de l'homme,

Vous osez seuls aux morts refuser des honneurs !

Que dis-je ? Vous craignez de montrer vos douleurs!

Sommes-nous dans ces jours de crime et d'esclavage?

Où de l'humanité prescrivant le langage,

Des tyrans dans nos yeux faisaient rentrer nos pleurs, 75

Où tous les sentiments se cachaient dans les cœurs ?

Le frère alors fuyait les obsèques d'un frère ;

Le fils suivait de loin le cercueil de son père :

On n'osait escorter que le char des bourreaux ;

La pompe de la mort n'était qu'aux échafauds !

Si de ce règne affreux, l'opprobre enfin s'efface,

Dans nos convois encor pourquoi m'offrir sa trace?

Quel Français sans gémir peut voir leur nudité ?

Craint-on qu'au sein des jeux un moment attristé

L'homme heureux, de la mort reconnaissant l'empire, 85

Ne s'aperçoive trop que son semblable expire?

$\mathrm{Eh}$ ! Ce corps à la terre indignement rendu,

Comme un vil animal dans les champs étendu,

Peut-être est-ce un savant dont le vaste génie

Par d'utiles travaux éclaira sa patrie !

90

Peut-être est-ce un ami des mortels malheureux !

Quel contraste! jaloux de prodiguer pour eux

De ses soins, de ses dons l'active bienfaisance,

Tous les infortunés recherchaient sa présence ;

Vivant, de sa maison ils assiégeaient le seuil ;

Mort, ils n'osent, hélas ! Entourer son cercueil !

«Pourquoi, me direz-vous, des honneurs funéraires?

Cette loi, que jadis établit chez nos pères

Un culte fanatique, et sans force aujourd'hui,

Sur nos bords éclairés doit tomber avec lui. »

$\mathrm{Ah}$ ! laissez ce langage au profane athéisme ;

La sensibilité n'est pas le fanatisme :
45

Elevar se siente el alma.

Testigos las artes son;

De muertos, que el mundo ensalza,

Ora el vivo metal,

Ora en la piedra animada,

$\mathrm{Al}$ universo encantado

Presentan la semejanza

Mas esto no satisface

Al corazón que los ama ;

Sus bustos $;$ ay ! ; No son ellos !

Ellos, sus restos, nuestra ansia

55 Quiere hallar, y de sus tumbas

Al pie meditar le agrada.

Allí del recogimiento

Gozando la dulce calma,

Al mismo tiempo lecciones

$\mathrm{Y}$ tristes lágrimas se hallan.

Parece que del profundo

De sus losas celebradas

Se oye una voz que nos dice :

" Alcanzad, como ellos, fama »

¡ He aquí las ilusiones ,

A nuestro anhelo robadas!

O tú por quien todavía

Llora la patria enlutada,

Consuélate sin embargo ;

$\mathrm{Si}$ en triste destierro vagan

Lejos de su monumento

Tus reliquias mutiladas,

De tu féretro arrojado,

Empero no de tus palmas,

Grabado nuestra memoria

Y nuestro pecho te guardan.

Allí tu mortal despojo

Su seguridad alcanza ;

Allí se han reedificado

Tu mausoleo y tu aras ;

Y sublevando los siglos

Contra la perversa raza,

Aun de sus viles ultrajes

Inmortalizas la infamia.

Mas ; qué nuevo crimen hiere

Mi vista escandalizada!

¡ Un cadáver conducido

Sobre espaldas mercenarias,

Sin comitiva, sin duelo

Solitario se adelanta !

¡ Así entregamos el hombre

De la tierra a las entrañas !

Naturaleza, amistad

Y el amor, acompañaban

En otro tiempo su polvo

A su postrimer morada,

Y su dolor consolante

En tributo le llevaran ;

Ágora, empero, inhumado

Sin las pompas funerarias,

Qué a los mortales, dormidos

En la tumba, circundaban,

Se diría, que ni un deudo

Ni un solo amigo contaba.

¿ Ha perdido sus derechos

Perdiendo la lumbre clara?

¿ No le debemos respeto ?

En la riveras, que baña

El Nilo, un celo industrioso, 
De la religion gardons l'humanité.

Barbares, qui des morts bravez la majesté,

Éloignez, s'il faut, ces ornements, ces prêtres

Dont le faste à la tombe escortait nos ancêtres ;

-Mais a

Mais appelez, du moins, autour de nos débris

Et la douleur d'un frère, et les larmes d'un fils.

C'est le juste tribut où nos mânes prétendent ;

C'est le culte du cœur que surtout ils attendent.

Mais si vous leur rendez cette pompe du deuil,

Oserez-vous encor reléguer un cercueil

Aux lieux où, nous plongeant dans les mêmes abymes,

La mort confusément entasse ses victimes ?

O trop coupable effet d'un usage odieux !

Auprès des scélérats gît l'homme vertueux !

Dans le même sépulcre indigné de descendre,

A leur cendre il frémit d'associer sa cendre.

Du juste qui n'est plus respectez le repos ;

Du juste et des méchants séparez les tombeaux.

Loin sans doute l'orgueil du pompeux mausolée,

Qui distinguait des grands la poussière isolée ;

Mais qu'au moins dans les bois un monument dressé

Dise au fils : C'est ici que ton père est placé.

Les bois ! ils sont des morts le véritable asile ;

Là, donnez à chacun un bocage tranquille ;

Couvrez de leur nom seul humble monument ;

De l'urne d'un héros son nom est l'ornement.

Ces dômes de verdure où le calme respire,

Le ruisseau qui gémit, et le vent qui soupire,

La lune dont l'éclat, doux ami des regrets,

Est plus mélancolique au milieu des forêts ;

Tous ces objets, que cherche une âme solitaire,

Prêteront aux tombeaux un nouveau caractère.

Par ce charme, appelé vers leurs restes flétris,

Nous viendrons y pleurer ceux qui nous ont chéris ;

Nous croirons voir planer leurs ombres attentives ;

Nous croirons qu'aux soupirs de nos âmes plaintives

Répondent de leurs voix les accents douloureux,

Dans la voix des zéphyrs gémissant autour d'eux.

Que la sage Helvétie offre un touchant exemple !

Lorsqu'un mortel n'est plus, là les siens, près du temple,

Vont déposer sa cendre en un bocage épais ;

Y plantent des lilas, des roses, des œillets,

Arrosent chaque jour leurs tiges abreuvées ;

Il semble qu'en ces fleurs, par leurs mains cultivées

Ils raniment l'objet près d'elles inhumé,

Et respirent son âme en leur souffle embaumé.

Comme eux à nos regrets sachons prêter des charmes ;

Rendons les fleurs, les bois, confidents de nos larmes ; 150

Dans les fleurs, dans les bois, du sort trompant les coups,

Nos parents reviendront converser avec nous.

Tout rendra leur aspect à notre âme apaisée ;

Les champs peuplés par eux deviendront l'Élysée.

Et les tristes humains, près de faire à leur tour

Ce voyage effrayant, qui n'a point de retour,

Comptant sur les honneurs dont la mort est suivie ;

$\mathrm{Ne}$ croiront pas sortir tous entiers de la vie ;

Et, par ce doux espoir en mourant ranimés,
Perpetuados á las miradas

Con incorruptible esencia,

Ya la madre arrebatada

Ya la consorte querida,

Sabía engañar la parca,

$Y$ fingir la vida. El Tiber

Y el Eurotas presentaban

Sobre las tumbas, ofrendas,

Hecatombes y plegarias.

¿Qué digo ? El salvaje mismo

De alma implacable, inhumana,

Siempre dio á su semejante

Un poco de tierra. O Francia

Tú , pueblo civilizado,

En esta edad celebrada,

En que Montesquieu,

Rousseau,

De Ferney el Patriarca,

Y Raynal, en sabias obras

Llenas de Grecia y de Italia,

La dignidad han mostrado

Del hombre á la especie

humana,

¡ Tu sola este honor reúsas

Al que la muerte arrebata!

Aun haces mas ; ; descubrir

Temes tus penas amargas!

¿ Lucen los días de crimen

Y esclavitud por desgracia,

En que de alma humanidad

Proscribiendo las palabras,

Entrar hacia en los ojos

Nuestro llanto voz tirana?

¿En que todo sentimiento

En los pechos se encerraba?

Las exequias de un hermano

Huía fraterna planta ;

Seguía el hijo de lejos

Del padre las tristes andas :

De los verdugos el carro

Solo acompañar se osaba ;

Solo al cadalso las pompas

De la muerte se guardaban.

$\mathrm{Si}$ de este odioso reinado

Se ha borrado al fin la infamia,

Nuestros entierros, ¿ porque

Su memoria nos retratan?

¿Q Quien mira su desnudez,

Y lágrimas no derrama?

$i$ Tememos, que entre sus dichas,

Turbada un momento su alma,

El feliz, reconociendo

El imperio de la parca,

No se aperciba, que el hombre

Cede á su segur avara ?

Esta ceniza á la tierra,

Indignamente entregada,

Cual la de un vil animal,

Extendida en la campaña,

Es la ceniza de un sabio.

Tal vez, cuya mente vasta

Con tareas provechosas

Al patrio suelo dio fama.

Tal vez es la de un amigo

De los tristes i Suerte extraña!

Celoso él de prodigarlos 


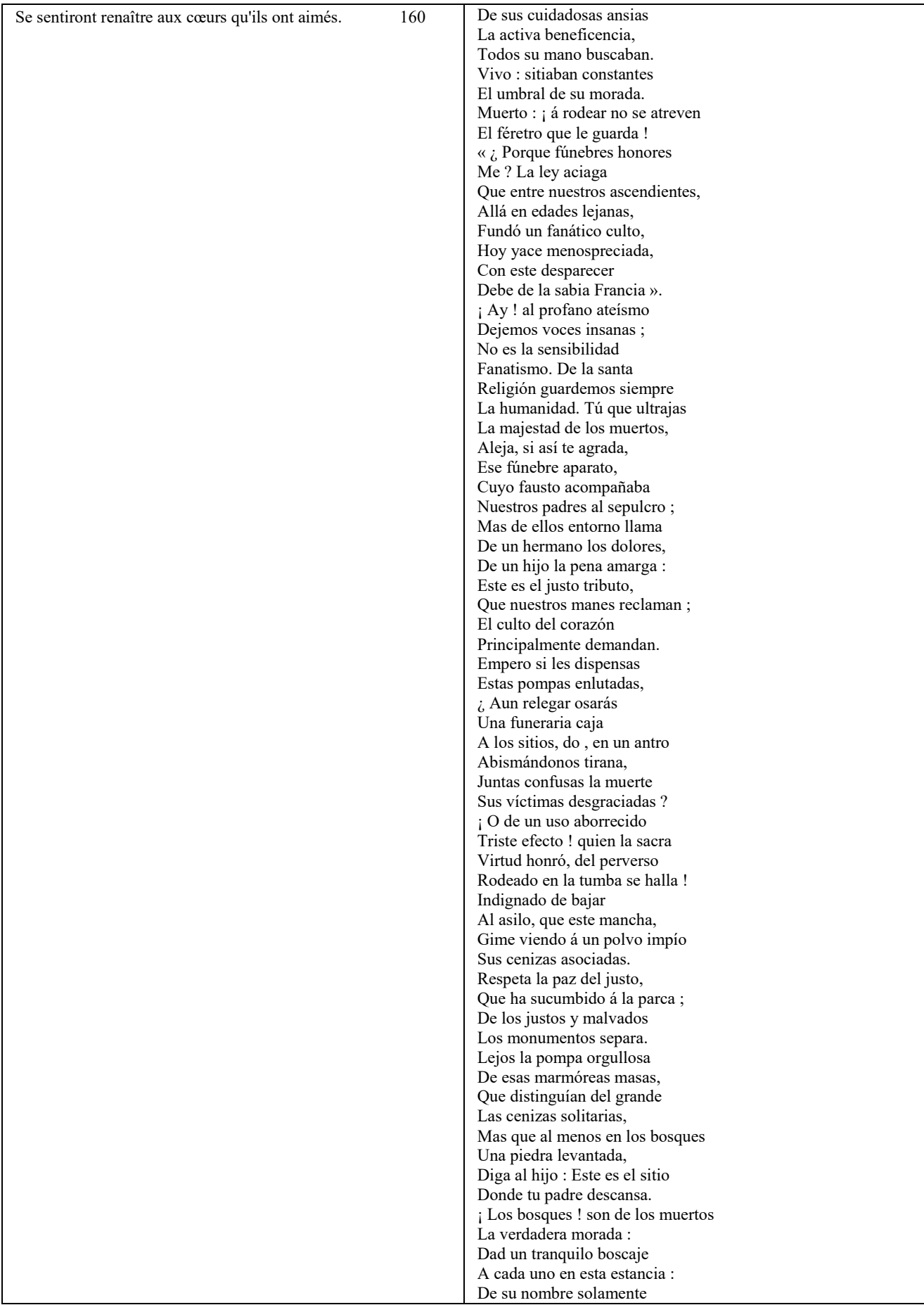




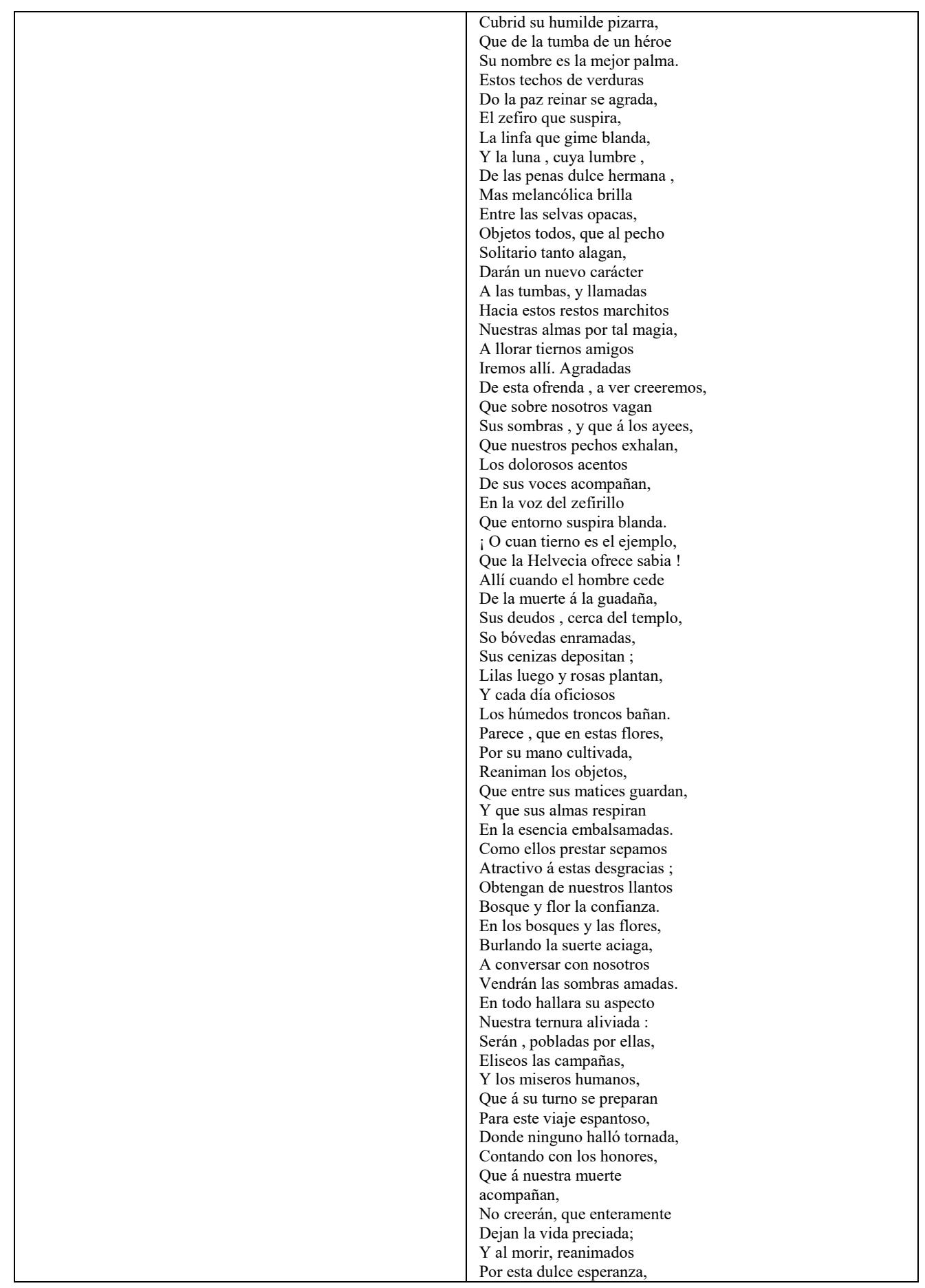


La différence de longueur entre le texte en langue originale et le texte en langue cible est la première chose que nous remarquons. L'élégie de Legouvé se compose de 160 vers, tandis que la traduction de Pérez de Camino est un poème de 312 vers. Il s'agit d'un élément très révélateur que le traducteur justifie par cette maxime : "Fidélité sans servitude". Cette ligne générale est celle que Pérez de Camino maintient dans toutes ses traductions et, à propos de traductions, il en distingue trois types. Premièrement, celle des partisans de la traduction littérale ou "travail servile", pour reprendre ses termes, aussi bien celle de ceux qui sont obligés à traduire tous les mots que celle de ceux qui rendent tous les mots et toute la métrique. Deuxièmement, il y a à l'autre extrême ceux qui se permettent toutes les libertés et deviennent ainsi, pour beaucoup d'entre eux, plus que des traducteurs, des imitateurs ou recréateurs d'œuvres. Finalement, appartiennent au troisième type ceux qui ne se situent ni dans un extrême ni dans l'autre, partisans de «la fidélité sans servitude et sans licence»; ceux-ci n'ont pas besoin de rendre le mot à mot ni de restituer les vers dans des mètres du même type, ils essayent de chercher les termes les plus justes et de trouver dans les langues modernes une métrique analogue à celle utilisée par les anciens. Pour Pérez de Camino, ces traducteurs sont ceux qui pourraient s'approcher le plus d'une perfection qui, de toute manière, est impossible en traduction.

Dans sa traduction de La Sépulture, Pérez de Camino a fréquemment recours au vers libre, qui est la seule manière de rester fidèle à la notion de traduction «sans servitude et sans licence», mais il ne cache pas non plus l'influence de la langue et de la culture françaises sur sa traduction. Il convient de souligner qu'il a passé presque autant d'années en France qu'en Espagne, puisqu'il avait trente ans quand il a traversé la frontière pour ne plus revenir dans son pays natal ensuite. En effet, il conserve tout au long de la traduction les usages de la ponctuation propres au français de l'époque. La tâche de traduire, notamment des œuvres littéraires et poétiques, est toujours une tâche ardue et d'une grande responsabilité, dans laquelle le traducteur fait des équilibres constants entre deux univers lexicologiques et morphosyntaxiques. Ces domaines sont ceux qui absorbent le plus grand effort et concentrent une grande partie de l'attention. Il y a cependant un aspect de la traduction qui n'est pas moins important, il s'agit des questions ortho-typographiques. Traduire une œuvre poétique implique également de respecter le rythme et la cadence de la langue d'arrivée, ses particularités orthographiques ainsi que ses normes de typographie qui, souvent, même si elles paraissent capricieuses, reflètent la manière particulière de comprendre le monde de ceux qui parlent cette langue. Pérez de Camino conserve les usages de la ponctuation propres au français de l'époque non seulement dans la traduction du texte poétique mais aussi dans tout ce qu'il écrit en langue espagnole, démontrant ainsi qu'il est le poète de style français «afrancesado» dont nous parlions au début. Ces éléments alourdissent et ralentissent parfois, le texte traduit. Pérez de Camino double presque les vers de Legouvé comme s'il avait fait une paraphrase des vers en langue originale. Pour l'auteur espagnol, traduire sans servitude signifie qu'il faut rester fidèle au texte original sans, pour autant, plaquer le rythme du vers ou le sens de chaque mot. La fidélité au texte original en poésie demeure une chimère pour les traducteurs. La traduction fidèle ne doit pas être littérale - correspondance difficile à trouver - mais, tel que Valentín García Yebra le signale dans sa Teoría y práctica de la traducción (1982: 38-39) : «La regla de oro para toda traducción es, a mi 
juicio, decir todo lo que dice el original, no decir nada que el original no diga, y decirlo todo con la corrección y naturalidad que permita la lengua a la que se traduce». C'est-à-dire, en gardant le registre contenu dans le texte original, afin de produire chez le lecteur lisant dans l'autre langue le même effet - ou le plus proche - de celui que l'auteur a voulu produire chez le lecteur lisant dans la langue originale. En d'autres termes, un texte poétique, malgré les difficultés de sa traduction, doit être maintenu le plus possible dans sa nature.

Si l'on cherche le mot "traduire" dans le dictionnaire, que trouvons-nous ?

«Che cosa vuol dire tradurre?», Ce sont les premiers mots d'Umberto Eco dans son livre Dire quasi la stessa cosa.

La prima e consolante risposta vorrebbe essere: dire la stessa cosa in un'altra lingua. Se non fosse che, in primo luogo, noi abbiamo molti problemi a stabilire cosa significhi "dire la stessa cosa", e non lo sappiamo bene per tutte quelle operazioni che chiamiamo parafrasi, definizione, spiegazione, riformulazione, per non parlare delle pretese sostituzioni sinonimiche (2004: 9).

Dans la nouvelle édition du Dictionnaire de l'Académie française, traduire signifie : «transposer un texte d'une langue dans une autre»; dans le Diccionario de la Real Academia de la lengua española, la première signification du mot traduire est : «expresar en una lengua lo que está escrito o se ha expresado antes en otra», tandis que dans le Webster New Collegiate Dictionary, parmi les définitions de "to translate" nous trouvons : to transfer or turn from one set of symbols into another. Nous ajouterions à toutes ces définitions correctes une idée déjà acceptée par les experts, selon laquelle une traduction ne se limite pas au seul transfert du contenu linguistique d'une langue dans une autre, mais aussi des contenus culturels. Chaque comparaison linguistique est aussi une comparaison culturelle parce que nous ne pouvons pas séparer le concept de langue de celui de culture. La traduction est le moment final d'une étude approfondie, d'une recherche détaillée aussi bien au niveau linguistique que culturel, dans une tentative de se rapprocher de la weltanschauung de l'auteur traduit et de découvrir ses secrets et les exprimer dans la traduction. La véritable traduction est transparente, elle ne cache pas le texte original ni ne le dilate, il faut choisir les mots un par un et recréer les mêmes sensations dans une culture différente.

\section{CONCLUSIONS}

La Sepultura de Pérez de Camino revendique la fidélité dans la traduction, entendue non pas comme un transfer ad litteram, mais comme une transposition d'essais, qui reproduit les valeurs du texte en langue originale. Nonobstant, ce modus operandi est la cause d'omissions et/ou d'apports inutiles de parties du texte et d'un artifice stylistique et syntaxique qui fait que la traduction n'est pas fluide. Dans La Sepultura nous ne notons pas d'omissions, bien au contraire, sa longueur injustifiée entraîne la perte du point de rencontre entre les deux textes.

Il ne faut pas oublier que La Sepultura de Pérez de Camino est le seul témoignage de l'élégie de Legouvé en Espagne et qu'elle date de 1822. C'est une traduction qui aurait besoin d'une nouvelle version, plus légère et plus simple, mais où légèreté et simplicité ne soient pas synonymes de pauvreté de style, mais du contraire. Il faudrait essayer de l'uniformiser et de l'harmoniser avec le texte original en évoquant dans la langue espagnole ces images et sons qui ont fait de Gabriel Marie Legouvé une des plus belles voix de la littérature sépulcrale européenne. L'exemple de traduction qui suit, qui est le résultat d'une réflexion personnelle sur la traduction de Pérez de Camino, est un projet encore in fieri : 
l'élaboration d'une traduction moderne, respectueuse de la tradition et qui cherche la fusion entre traduction et interprétation. Il s'agit des 25 premiers vers de l'élegie de Legouvé:

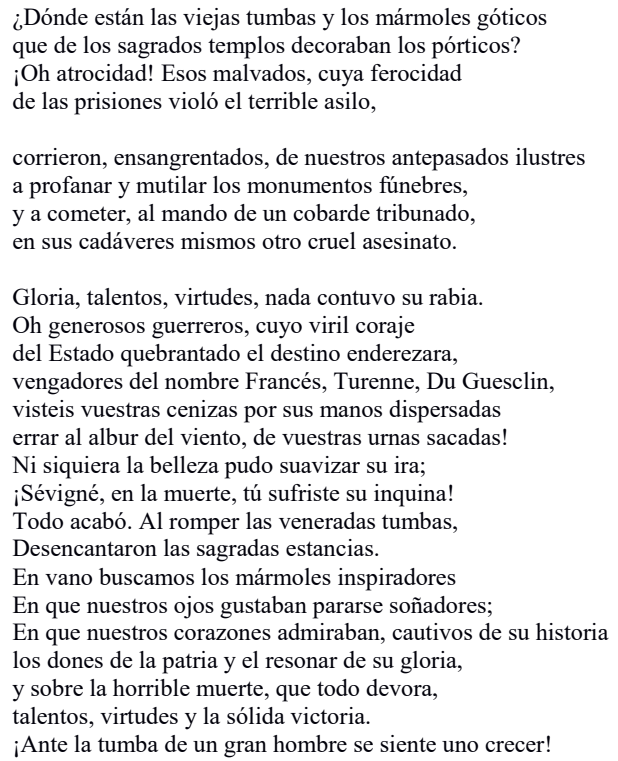

Les vers traduits conservent, dans la limite du possible, la structure, le lexique, les images et le rythme du texte français. À cet égard, et dans le but de préserver le rythme du vers français, je voudrais souligner quelques points de ma traduction à l'espagnol. Premièrement, pour conserver en espagnol le couple français formé par antiques / portiques (vv. 1-2), j'ai écarté la solution offerte par Pérez de Camino antiguos / pórticos -qui fait perdre complètement le rythme qu'apportaient les vers originaux - j'ai remplacé l'adjectif antiguos par góticos - qui sont également anciens - afin de permettre la rime avec pórticos. Deuxièmement, et tout en continuant avec le rythme, afin de conserver en espagnol le couple français tribunat / assassinat (vv. 7-8), j'ai changé le mot tribuno de Pérez de Camino par tribunado qui rime avec asesinato. Troisièmement, le duo inspirants / longtemps (vv. 19-20), que Pérez de Camino traduit littéralement, je l'ai traduit en lui donnant un ton plus romantique et en cherchant dans le lexique propre de la poésie sépulcrale : inspiradores / soñadores, où soñadores est la traduction du binôme s'arrêter longtemps.

Ce sont seulement quelques exemples qui ouvrent la porte à une étude contrastive - traductologique aux dimensions plus larges et qui ne veut à aucun moment enlever de son poids ou de son importance à la traduction de Manuel Norberto Pérez de Camino. Ce dernier, effectivement, continue d'être la seule référence tangible pour le chercheur dans le domaine de la poésie sépulcrale espagnole et en langue espagnole.

\section{BIBLIOGRAPHIE}

Abellán, José Luis (1973): “Manuel Pérez de Camino, poeta y pensador”. Bulletin Hispanique, tome LXXV: 132-168. 
Alonso MARTínez, Manuel (1874): "Prólogo", in Elegías de Tíbulo, traduites au castillan par M. N. Pérez de Camino. Madrid, imprimiere Julián Peña.

ECO, Umberto (2004) : Dire quasi la stessa cosa. Milano: Bompiani.

GARCÍA YEBRA, Valentín (1982): Teoría y práctica de la traducción. Madrid: Gredos, tome II.

Hurtado, Juan y GonZÁlez PAlEncia, Ángel (1943): Historia de la literatura española. Madrid: S.A.E.T.A.

LEgOUVE, Gabriel Marie (1801): Euvres complètes. París: A. Burdin.

MARANTONio SGUERzo, Elsa (1976): Evoluzione storico giuridica dell'istituto della sepoltura. Milano: Giuffrè.

MENÉndeZ Pelayo, Marcelino (1962): Historia de las ideas estéticas en España. Madrid: Anaya, tome III.

MenÉndez Pelayo, Marcelino (1965): Historia de los heterodoxos españoles. Madrid: Anaya, tome VI.

PÉREZ DE CAMINO, Manuel Norberto (1822): El mérito de las mujeres, los recuerdos, la sepultura, la melancolía : poemas de Gabriel Legouvé, traducidos en versos castellanos por Don M. N. Pérez de Camino. Burdeos: Lawalle.

PÉREz GÁlvez, José (1997): El sistema funerario en el Derecho Español. Pamplona: Aranzadi.

RIVADENEYRA, Manuel (1871): Bibliotecas de autores españoles. Madrid: Rivadeneyra editeur.

RONCORONI, Angelo (1999) : Leggere lirica. Milano: Arcipelago.

TOLÍVAR, Leopoldo (1983): Dogma y realidad del derecho mortuorio español. Madrid: Instituto de estudios de administración local.

\subsection{Dictionaires consultés}

Dictionnaire de l'Académie française: $\underline{\text { www.atilf.fr }}$

Diccionario de la Real Academia de la Lengua Española (DRAE): www.rae.es

New Edition of Marriam-Webster's Collegiate Dictionary, Springfield, 2003. 